\section{ABSTRACT OF A CLINICAL LECTURE} ON

\section{A CASE OF SPINA BIFIDA CURED BY INJECTION.}

BY JAMES MORTON, M.D.,

Surgeon and Clinical Lecturer, Glasgow Royal Infirmary.

JoHn KelLy, aged two months, was admitted into Ward 17 of the Glasgow Royal Infirmary on October 2nd, $187 \mathrm{I}$. This child had a tumour opposite the upper lumbar vertebrae, globular in shape, of about the size of a small orange, and presenting the usual appearances of a case of spina bifida. Its covering was thin, so that it was quite translucent; and at the child's birth it was about half its present size. The mother stated that the tumour felt harder and fuller when the child cried. Moderate pressure upon it did not cause much inconvenience or suffering; and the child had never had convulsions, and seemed in all other respects quite healthy; the fontanelles being neither more open nor more full than usual at the same age.

On October I2th, the tumour was punctured with a grooved needle, and rather more than half of its contents-a pale straw-coloured fluidwas removed. It was then covered with lint dipped in oil, and over this cotton-wool ; and, to fix the whole and afford a slight pressure, a broad elastic band was passed round the waist of the child. The sac speedily refilled, and on the 18 th was punctured a second time; and again it refilled.

On October $24^{\text {th }}$, the tumour had regained nearly its former size; and to-day it was punctured with a trocar and cannula of moderate size. After it had been half-emptied, a small portion-probably nearly half a drachm-of the following solution was injected : R Iodi gr. $x$; potass. iodidi 3 ss ; glycerini $\tilde{3}$ i.

On October 26 th, the child continued well, having no bad symptoms, and being only a little "fractious". In the swelling, a portion of a soft solid substance could be felt, rather to one side.

On November 2 nd, the mother was allowed to take the child home for a day or two ; and on the 6th she returned to the Infirmary, when it was evident that some degree of refilling had taken place. The tumour was again injected with a small portion of the same solution. How much actually entered the sac, it was not possible to say. The child, however, continued quite as well as before ; and on Novemher 23rd he was brought up and shown to the clinical class, when the tumour was found to be smaller and harder than formerly, without any appearance of re-distension with fluid.

On December 7 th, the child was again shown at the hospital ; and now the swelling presented the appearance of a shrivelled bag of skin, darker in colour than the adjacent integument, somewhat resembling a corrugated scrotum, and affording reason to believe that there was now complete closure of the opening in the spinal membranes. The health of the child was perfect, his mother affirming that he never was so well and comfortable as he was now.

On January 8 th, 1872, the child was again brought to the hospital, merely to show that he continued quite well.*

REMARKS. - In commenting on this case before the clinical class, a short account was given of the usual appearances presented by such tumours, and the ordinary coverings and contents were noted. Reference was also made to the deficiency in development of the osseous portion of the spinal canal, as the origin of the name, which of itself conveys no idea of the importance of the contents; and remarks were further made to the following effect. It is in reality a local dropsy, consisting of a collection of serum within the serous covering of the spinal cord; and, too often for the safety of the patient, there is also a portion of the nerve-structure of the cord, which has left its proper line, and lies extended under the serous lining of the sac, just under the skin, running, as it were, round or half round the circumference of the swelling, which is often globular, as in this instance. It is this condition chiefly which renders interference with such cases hazardous, and which presents to the mind of the surgeon the possibi. lity of the nervous matter of the cord being so disturbed as to lead to very violent and dangerous manifestations of the effects of irritation of such tissues, in the form of convulsions, which not seldom terminate fatally. Even when left to nature, the rule seems to be, that the subjects of such malformations die, and that early-within the first or second year. To this, it is well known, there are exceptions; still it is

* This patient was shown to the members of the Medico-Chirurgical Society at the meeting on the ist February last. the rule. Attempts are usually made by relatives, under professional advice, to protect the part by the use of hollow cup-like shields, lined with cotton, and most carefully attended to ; but the result is disappointment, so far as life is concerned. These tumours are far more common in the lumbo-sacral region than in all the other portions of the spine put together; and this is an example of one in the lumbar region.

TREATMENT.-Allusion has just been made to the palliative or protective treatment, as we may venture to call it ; and we now turn our attention to the modes of attempting the radical cure, and give shortly our reasons for adopting the plan which has been so fortunate in the present instance. Though it may be true that active surgical interference usually hastens death, yet cases have recovered after various kinds of treatment. The presence of the cord, and the free communication with the serous covering of the spine and brain, are the chief obstacles to success. Inflammation of the cord or its membranes, and gangrene of the cord, causing paralysis, may occur, speedily ending in death. Even when left untouclied, the skin often ulcerates, the sac bursts, and palsy or convulsions cause death. A spontaneous cure has been known to take place, the orifice of communication having closed, the tumour becoming a closed cyst, and remaining innocuous, or it may be removed. Rupture of the sac has even been followed by recovery. Three modes of interference present themselves-injection, ligature, and excision; and of these three modes, it may be said that each of them has been effected by a variety of means and appliances, which it is not my purpose to enumerate at present. Suffice it to say, that I resolved to adopt the first of these, as, in my opinion, the least dangerous of the three. It will be noticed that twice I punctured the sac with a grooved needle, and drew off a considerable quantity of the fluid. These may be called tentative measures, to ascertain whether the membranes could be pierced with safety; and, no suffering or derangement of function having followed, I felt encouraged to use an iodine solution. In forming this solution, I resolved to use glycerine as the solvent; for this reason, that it is a fluid of less diffusibility than a spirituous or even a watery solution. Not that I for a moment imagine that it could not be conveyed along the serous cavity or the serous lining of the spine; still, to my mind, it offered one element of greater security. The strength of the solution may be noted ; but then it must be observed that, in using it, the sac was purposely only half-emptied of its serum when the iodine solution was injected; and of the latter only about half a drachm was actually injected, so that the then ioduretted contents of the sac presented a very dilute solution. When the repetition of the injection was made, somewhat more of the iodine fluid was used; but part of it escaped and was lost, so that, as the report states, it was not possible to estimate correctly the amount retained. A degree of solidification followed the first injection; and after the second it became complete, and now remains so, the part admitting of ordinary handling without inconvenience or discomfort to the child.

A line of treatment very similar to this has been followed by several of the American surgeors, and with instances of success; but statements are discordant in regard to the proportion which the successes bear to the failures. Several British surgeons have also operated successfully in a limited number of cases and by various methods, but most frequently by injection with iodine as the coagulating or stimulating agent ; but I am not aware of glycerine having been used as the solvent on any previous occasion, and I may indulge the hope that others may be induced to try it.

From what I have seen of such cases, it is my belief that the fluid should not be allowed to be drained completely from the sac : this leads to fatal results; and, where a puncture has been made, the aperture, however minute, may be closed and guarded by a layer or layers of collodion, especially when subsequent oozing is feared or perceived.

\section{OBSERVATIONS ON MEGRIM OR SICK-HEADACHE.}

\section{BY EDIVARD LIVEING, M.D.}

IT is now just two years since I sent to the Professor of Medicine at Cambridge a thesis on the subject of "Megrim or Sick-Headache", which was publicly read in the school there on the th of April, is7o. My paper opened with a detailed narrative of a number of cases selected from my own practice or recorded by others, and was intended to show that a variety of nervous affections hitherto treated of for the most part in an isolated way, under such names as hemicrania, migraine, sickheadache, biliousness, blind-headache, suffusio dimidians, hemiopia, neuralgie ophthalmique, and so forth, are really only different phases o the same malady, or members of one pathological family, which 
might with advantage be included under some common name such as megrim.

These cases were so arranged as to exhibit the transitions from the simpler to the more complex forms of the seizure. They comprised instances where the affection was represented in most attacks by a single feature, such as a violent paroxysmal headache of a culminating character, either hemicranial or bilateral ; an attack of nausea, vomiting, and prostration, very like those of sea-sickness ; or a transient disorder of vision, like the well-known half-blindness of Dr. Wollaston, in which " he suddenly found he could see but half the face of a man whom he met; and it was the same with respect to every object he looked at. In attempting to read the name JoHnson over a door, he saw only soN"; or the same with the addition of certain spectral appearances. There were also instances where all these phenomena occurred in succession in the same case, often reaching a high degree of intensity ; and still more complex forms in which were superadded a numbness and tingling, generally having a centripetal progress towards the head and neck from the fingers, or fingers and toes of one side; not unfrequently a transient form of aphasia, or difficulty of utterance ; and occasionally a confusion of ideas or failure of memory of a similar transient character.

The real affinity of these different seizures was further shown by such facts as the following. An habitual sufferer from one of the simpler forms of the malady would have his attacks replaced from time to time by the graver and more complicated paroxysm; or, of different members of the same family, one would suffer from one form and one from another. Many of the characteristic phenomena were illustrated in considerable detail in the selected cases, and numerous extracts were supplied from both English and Continental writers in confirmation or illustration of various points. In particular, I may mention the amau. rosis, and the singular zigzag scintillating spectrum described by many patients as following the obliteration of a portion of the visual field. Among these I quoted the celebrated Dr. Fothergill's account of his own case, in which he describes the spectral appearance as " surrounded by luminous angles, like those of a fortification". This corresponds with the elaborate description recently given by Dr. Airy, in a paper read before the Royal Society in 1870 , of what he has himself witnessed in his own person; but the extract from Fothergill was not communicated to him until after the reading of my thesis, and the use of the term "trichopsia" in his interesting paper was therefore an undesigned coincidence, strongly confirming the identity of the appearance in both individuals.

The strictly aphasic character of the disorder of speech in a certain number of cases was also shown, and the remark of one of my patient was quoted-that he could not tell why, but he was for the time utterly unable to pronounce the words he had in his mind, and that if he attempted to do so he should utter some which had no meaning, or which he did not intend. As a supplement to this portion of the history of megrim, I pointed out, in the Thruston speech, delivered in Caius College in May last year, "On Disorders of the Faculty of Speech", that a singular correspondence might be observed between the phenomena of this purely functional malady and those of some cases of brain-disease, in the almost exclusive association of aphasic symptoms with hemiplegia of the right side. Megrim, as its name implies, is very frequently an unilateral affection; and I observe that the aphasic cases have been, so far as my analysis extends, and subject to future correction, exclusively those in which the other phenomena-more particularly the numbness and tingling - have been either confined to the right side or bilateral.

The maintenance of a tolerably constant and definite order and succession among the leading symptoms, whenever two or more were present, was also pointed out. Thus the visual phenomena are mostly initial, and always take precedence of the pain, while the vomiting is the last in the series.

Addressing myself to the affirmative proposition of the thesis-that the malady is essentially one of the nervous system, mostly hereditary, and of which the paroxysms may be described as nerve-storms-I exhibited an analysis of upwards of fifty cases, in which all the principal features of the disorder, as well as the special phenomena of the paroxysm, were set out in a tabular form. These showed briefly: I. Certain limitations as to age, both with respect to commencement and termination. 2. A preponderance-at least as regards some varieties of the affection-in one sex. 3. The very powerful influence of a hereditary disposition in giving rise to this malady, which above all others is a "family complaint". 4. The strictly paroxysmal nature of the seizures and freedom of the intervals; the attacks exhibiting an approach to periodicity in their returns in most instances, but with considerable variation in the length of the intervals in different cases, the same interval, however, being approximately maintained in the same individual ; thus weekly, monthly, and bi-monthly periods are com. mon : closely connected with this is the immunity secured to the patient for a certain period after a severe seizure, and the aggravation of the next paroxysm if perchance a double interval should have elapsed. 5. The influence of accessory and exciting causes in dotermining attacks in subordination to this approximate periodicity; such influence; being operative-at least in the majority of sufferers-only at or near the time of spontaneous recurrence. Such are various forms of peripheral irritation, and certain states of the system ; more particularly gastric irritation; powerful impressions on the sensorium, as by the combined sights, sounds, and general excitement of public spectacles and assemblies ; ovarian irritation and catamenial periods; over-taxing the sight on small objects, or straining after accommodation by the asthenopic or hypermetropic ; strong odours ; and so forth ; and perhaps more certainly influential than any of them-mental emotion and excessive brain-work.

The various phenomena of the paroxysm were also exhibited in their natural succession, relative frequency, unilateral or bilateral character, termination, and after-effects.

All these features of the malady were then compared with those of the great natural family of Neuroses, represented by such affections as spasmodic asthma, epilepsy, angina pectoris, paroxysmal insanity, and the like; and it was shown, as I venture to think conclusively, that none of these have a better claim to that fellowship, or exhibit a more typically neurosal character, than megrim, as identified in the previous part of the thesis. The last link of confirmation was added by showing that attacks of megrim are sometimes replaced in the same individual by other neurosal seizures; as, for example, epilepsy or mania transitoria, although the rule is, that the same type of paroxysm is maintained throughout life. On the other hand, such transformation in the course of hereditary transmission is sufficiently common : thus a parent suffers from typical migraine, one of his children from the same malady, another from epilepsy, and a third perhaps from chorea.

I next discussed some of the prevalent theories as to the origin and cause of these seizures; and more particularly the "bilious" theory of Fothergill and of the general public, which I endeavoured to show was a legitimate descendant of the ancient humoral pathology; and also the "gastric" or sympathetic theory, which supposes the affection of the head to be merely symptomatic of gastric irritation, and which, although containing a measure of truth, is quite inadequate to explain the clinical history of the malady when viewed as a whole.

The discussion of the theory of a "determination of blood" to the head-at one time very much in fashion to explain such seizures-as well as the modern vaso-motor hypothesis, more particularly that form of it put forward by Du Bois Reymond, who supposes the affection to be one, not of the brain nor of the nerves of the brain, but of the "regio cilio-spinalis", and to consist essentially in a morbid excitation of the cervical sympathetic on one side, giving rise to a tetanic condi tion of the arteries on the same side of the head, which is the cause o pain, and to irregular contractions of the ophthalmic and vertebra arteries, producing oscillations of the blood-pressure in the visual apparatus and brain, causing the disorder of sight and the sickness-these were necessarily passed over for want of time. I may, however, take this occasion to say that, having had the opportunity of examining the fundus of the eye with the ophthalmoscope in one of my patients during the transition from the stage of blindness to that of pain, I was unable to notice any alteration or difference in the vascularity of the retina or optic entrance as compared with that of the same parts on the following day. On another occasion, I carefully compared the temporal arteries in a well-marked hemicranial paroxysm, but could discover no such increased rigidity of the one on the painful side as $\mathrm{Du}$ Bois Reymond describes.

In conclusion, I stated that, having regard to the almost exclusively sensory character of the phenomena (the only exception being the derangement of certain complex consensual movements like those of speech or manipulation) and the natural sequence they exhibit, and extending the argument used by Wollaston with respect to the affection of vision, that nothing but a disturbing cause situated behind the optic chiasma could explain the symmetrical affection of the visual field in each eye, so nothing but a like cause operating in the neighbourhood of their common origin along the sensory tract at the base of the encephalon could explain the successive implication of nerves so remote in their distribution as those of sight, the sentient nerves of the extremities, the trigeminus, and the pneumogastric ; and bearing also in mind that the cause must be of a kind to explain both the interruption of the ordinary transmission of objective impressions and their replacement by subjective ones, as well as the strictly paroxysmal and culminating character of the symptoms, no hypothesis seemed to me so well adapted to explain the phenomena as that of a nerve-storm traversing more or less of the sensory tract from the optic thalami to the ganglia 
of the vagus, or else radiating in the same tract from a focus in the neighbourhood of the quadrigeminal bodies. The analogy of epilepsy, which is supposed to have its point of departure in a tendency to explosive discharge in the motor tract of the medulla oblongata, lends support to this view.

I am not at all disposed to deny that disorders of local circulation occur in the course of megrim, and that the implication of the sympathetic may play an important part in their production; but I regard them among the least constant and regular of the phenomena, and certainly not as essential and as the cause of the rest.

\section{CASE OF POPLITEAL ANEURISM CURED BY VOLUNTARY FLEXION OF THE LEG IN ABOUT THIRTY-SIX HOURS. \\ By T. HOLMES, F.R.C.S., Surgeon to St. George's Hospital.}

I THINK the following case may be of some interest to the readers, as well as to the editor, of the BRITISH MEDICAL JOURNAL, as an example of the great ease with which consolidation can sometimes be procured in a popliteal aneurism by the simple method which was first introduced into practice by Mr. Ernest Hart. The merit of our editor in this respect is in no degree lessened by the fact that Sir W. Fergusson or his house-surgeon had made some attempt of a similar kind before, or that the same idea had occurred to Maunoir, of Geneva, who seems to have put it successfully into practice. Neither of these facts was known at the time, so that they could have had no influence on Mr. Hart's discovery. I took peculiar interest in the matter, having seen the original case in consultation with Mr. Hart; and it happens that I have been peculiarly fortunate in my experience of the method, this being the second case under my own care in which a cure has resulted in two days. My former case was reported in this Journal (vol. i, 1868, p. 585). In small recent aneurisms (such as both of these were), if complete flexion of the knee abolish, or almost abolish, the pulsation, the surgeon may entertain a very confident hope of effecting a cure by this most simple, easy, and painless treatment. Nor is there occasion generally for any inconvenient constraint. The patient, being a man of ordinary intelligence, can easily be shown the effect produced on the -pulsation by the bending of the leg; and if it be inconvenient to him to have the limb bandaged, it can be left lying on its outer side, on a couple of pillows, in a position of as complete flexion as he can bear ; and he may generally be trusted to change that position to a slight extent from time to time. Next day, if no impression have been made on the tumour, the muscles will have become somewhat accustomed to their position, and he will probably be able to bear more complete and more rigid confinement; but this, in the present instance, was unnecessary. It is well, I think, to keep up the flexion for a day or two after the apparent cure, since we know that pulsation may recur after it has completely disappeared. At the same time, I am under the impression that this recurrence is very improbable if the anastomosing articular vessels have been plainly felt to be enlarged. It is an interesting feature in this case, that this enlargement of the collateral arteries had gone on to such an extent, in less than forty-eight hours, as to render them plainly perceptible to the touch. The completely fluid condition of the contents of the aneurism on the patient's admission renders it tolerably certain that no enlargement could have taken place previously. I append notes of the case, which have been kindly furnished to me by $\mathrm{Mr}$. Stirling, my crmical clerk.

CASE.-John G., professional cricketer, aged 32, was admitted on February 21st, 1872, and transferred to Mr. Holmes's care on the 23rd, with a circumscribed pulsating tumour of the ham. He was playing at cricket at the end of August, when, on stooping very suddenly to pick up the ball, he felt a slight pain in the ham ; during the rest of the match, he was conscious of a sensation of weakness in that part. During a month following, he neither thought nor felt that there was anything amiss ; but at the end of that period, he noticed a "lump" in the ham, which was attended with some pain and weakness; these symptoms he had experienced a few days before he noticed the lump. He applied to the tumour some lotion given to him by a surgeon, which apparently made it larger and more painful. He then came to the hospital.

On admission, there was found to be a rather hard pulsating tumour, about the size of a walnut, in the upper and outer part of the right popliteal space. The pulsation was of a distensile character, and was entirely stopped by pressure on the right superficial or common femoral arteries ; and when either of these was compressed, it could be entirely emptied of its contents. There was a very loud bruit. On his ad- mission, the tumour was accompanied by considerable gnawing pain, but this symptom subsided after he had been quiet in bed for a day or two. The man had enjoyed fair health, but was a rather free drinker. He was sent to bed, and ordered to have an ounce and a half of acid cinchona draught three times a day.

February 23 rd. - On this patient being handed over to Mr. Holmes on February 23 rd, about 3 P.M., he caused the leg to be very strongly flexed and bandaged upon the thigh ; but this produced so much pain that the patient could only keep it in that position for an hour and a half. Subsequently he flexed it himself for as long as he could, relax. ing the flexion when he could no longer bear it.

February 24th. - His leg was again forcibly flexed, but he could not endure the constraint even so long as on the previous day. He therefore continued voluntary flexion tolerably regularly at intervals. On examination at 2 P.M., the bruit was found to have quite ceased, and the tumour appeared decidedly more solid, with the pulsation diminished.

February 25 th. -No pulsation or bruit was perceptible. The superior internal articular artery was felt to pulsate.

February 26th. - The tumour was still solid to the feel, without perceptible pulsation or bruit. The voluntary flexion had been continued fairly regularly, and was still to be carried on. The aneurism was very painful and tender to the touch. The articular artery was more perceptible.

February 27th. - Flexion was left off.

March Ist. - The tumour was hard, and had considerably diminished in size. There was no tenderness in the ham. He was allowed to get up.

March 4th. - He had been taking gentle exercise in the ward since the above date. The tumour remained hard, lessened in size, and without pain. He had been unable to straighten the leg since flexion was left off; but on each day a manifest improvement in that respect was visible; and on March 9th, the limb was nearly straight. He was. discharged as cured.

\section{HYDATIDS OF THE BRAIN : OPTIC NEURITIS,}

$$
\text { By T. M. EvaNS, EsQ., Hull. }
$$

In the JOURNAL of September 28th, 1867, I recorded some cases of thrombosis and embolism occurring at the Hull Infirmary, and amongst them one headed "Cerebral Embolism following Valvular Disease of the Heart," which afterwards proved to be a case of hydatids of the brain. Having just learned that this case has. been quoted by Dr. Hammond in his work on Diseases of the Nervous System, I send the accom. panying notes of the further progress of the case, which were read by me at a meeting of the local Branch of the Association in May 1868.

A lad aged 17 was admitted into the Infirmary in April 1867, suffering from headache, vomiting, confusion of vision, strabismus, and facial paralysis, with a loud mitral bruit, resulting from a recent attack of acute rheumatism; and these symptoms, together with the ophthalmo. scopic appearances to be presently described, were supposed to denote " some morbid condition within the cranium, which appeared to have its most easy and natural explanation in cerebral embolism-an opinion further supported by the perfect recovery of the patient". However, seven months afterwards he was readmitted with headache, increased dimness of vision, and a staggering gait, being unable to walk without assistance, and he gradually sank exhausted. At the post mortem examination, on removing the brain, much fluid ran from the subarach. noid space, and from the ventricle, which appeared distended-the arachnoid membrane being also thickened, and the brain generally con. gested ; and protruding from the under surface of the cerebellum on the left side was a mass of hydatids, forming a tumour of about two inches diameter, most of them collapsed, but some remaining full and transparent. During his last stay in the Infirmary, being confined to his bed, he was not examined with the ophthalmoscope, but the appearance of the fundus in his first illness was full of interest. I quote from the JoURNAL: "The retinal vessels were found much enlarged, and the veins very tortuous; the optic disc, of an intense red colour, not being distinguishable from the surrounding parts, except by the entrance of the vessels, the redness being chiefly due to a number of very fine vessels radiating from the centre." Dr. Hughlings Jackson recently remarked that optic neuritis indicates the presence of "coarse cerebral disease", by which is to be understood some tumour or other palpable affection; and this case corroborates his views.

In the previous May, a very interesting paper had appeared in the Medical Times and Gazette, headed "The Ophthalmoscope in Physicians" Practice," in which were recorded several cases occurring in the prac- 